\title{
Damping prediction of structures with bolted joints
}

\author{
Lothar Gaul* and Jens Becker \\ Institute of Applied and Experimental Mechanics, University of Stuttgart, Pfaffenwaldring 9, 70550 Stuttgart, \\ Germany
}

\begin{abstract}
Friction in joints significantly contributes to the observed overall damping of mechanical structures. Especially if the material damping is low, the frictional effects in joints and clamping boundary conditions dominate the structural damping. The damping and the stiffness of the structure are nonlinear functions of the system states and consequently of the excitation signal and amplitude. If these nonlinear effects should be incorporated in the design process, transient simulations must be employed in order to predict and analyze the damping for a given excitation, though they need excessive computation power due to the nonlinear constitutive laws and the high contact stiffnesses.

As one approach to alleviate transient simulations, the application of component mode synthesis (CMS) methods to structures with friction is investigated exploiting the linearity of the jointed substructures. The friction and the nonlinear normal contact is modeled by constitutive laws that are implemented in node-to-node finite elements. The necessary considerations for accurate damping prediction by the reduced models, the accuracy and the computational times for transient simulations are discussed. The developed model reduction techniques allow a strong reduction of the computation time which in turn makes it a promising tool for model updating and predictive parameter studies. As an application example, a beam-like structure with attached friction damper is investigated in simulations and the obtained numerical results after model updating are compared to experiments.
\end{abstract}

\section{Introduction}

Most real-world engineering mechanical structures contain nonlinear elements, e.g. play, nonlinear springs and stiffnesses, or joints with nonlinear contact and friction effects. It is known from experiments that friction, mostly microslip effects, in joints contribute significantly to the overall structural damping in metal structures $[6,7,13$, 14]. Due to the nonlinearities, analysis must be conducted costly in time domain or with special methods such as harmonic balance methods $[12,14,17]$. This is often avoided by using linear stiffness and damping models for harmonic analysis in frequency domain [1]. By this, it is exploited that most nonlinearities are located on interfaces connecting substructures for model order reduction therewith enabling transient analysis with fair cost for engineering mechanical structures. For example, this makes closed-loop simulations of semi-active controllers for friction dampers with controlled normal forces feasible [2].

Although many publications cover model reduction techniques for mechanical structures with isolated nonlinearities, e.g. using Krylov-mode based reduction [8] or using proper orthogonal decomposition (POD) methods [16], only few consider distributed nonlinearities. For the investigated beam-like structure with attached friction damper element for vibration damping, friction and contact nonlinearities act on the contact area between base structure and friction damper. Though the contact area is small compared to the overall structure dimensions, it can not assumed to be point-like.

\footnotetext{
*Corresponding author. E-mail: gaul@iam.uni-stuttgart.de.
} 


\subsection{Literature review}

In Gaul et al. [8], Krylov reduction methods are employed to efficiently simulate the dynamics of a truss structure as a typical lightweight construction. The truss is equipped with adaptive friction joints that can be semi-actively controlled to damp out vibrations excited by a snap-back of the truss tip. Model reduction is applied for the simulation of the closed-loop behavior and for the design of appropriate observers. In the first step of the model reduction, the dominant modes of the structure are determined, all others are truncated. Then, Krylov modes are determined for the linear transfer functions between every discrete adaptive joint model and the controlled variables and added to the reduction base. By this technique, a strong reduction of the computation time is achieved as long as not too many nonlinear joints have to be considered which would strongly increase the number of transfer functions that must be separately treated. A strong disadvantage of this approach is that the dynamics of the whole structure can not be recovered from the reduced solution, i.e. the interesting output variables must be already determined before the reduction step is conducted. Qu [15] investigates model reduction techniques for finite-element (FE) models with local nonlinearities. He proposes an iterative procedure to calculate reduced mass matrices from Guyan reduction that improves the accuracy at higher frequencies without adding additional DOFs. Witteven and Irschik [18] propose model reduction techniques specifically for structures with bolted joints. They model only the nonlinear normal contact in the bolted joints without consideration of frictional effects. Jalali et al. [9] considered two beams connected by a rotational joint with friction that is modeled by a discrete nonlinear joint model. Their dynamic model is based on the first bending mode obtained from a linear case. For vibrations close to the one considered mode, the joint model parameters are identified from experimental data. However, it is not tested if the chosen joint model and the identified parameters are able to capture the dynamical behavior at other resonance frequencies as well and the question how to replace the nonlinear elements by linear ones for the modal reduction is not discussed.

Segalman [16] proposed the use of eigenmodes in addition to special discontinuous global vectors (either eigenvector sensitivities or so-called Milman-Chu vectors) in order to strongly accelerate convergence of a Galerkin reduction method for model reduction of medium-scale mechanical systems with localized nonlinearities. The method is demonstrated for a 11 degrees of freedom (DOF) system with 1-D friction nonlinearities with the system energy time evolution as performance criteria, for which superior convergence is shown if the proposed discontinuous vectors are used. Kappagantu and Feeny [10,11] apply proper orthogonal decomposition (POD) methods to determine reduction bases from arbitrary time signals that are obtained from either measurements or simulations. However, such POD methods generally yield black-box models that give no insight into the internal physics and the reduction quality strongly depends on a representative selection of the excitation signals. Only the selected outputs can be recovered from the reduced model solution, which strongly restricts the range of applications of these methods.

In summary - to the opinion of the authors - there is a lack of research of model-driven reduction strategies for mechanical structures with friction that systematically exploits the separation of the overall structure into jointed substructures. Such strategies are investigated in the following and applied to efficiently predict the damping contribution of bolted joints and friction interfaces to the observed overall structural damping.

\subsection{Basic concepts of component mode synthesis}

Component Mode Synthesis (CMS) methods reduce the complexity of structural dynamic models leading to reduced simulation time and reduced memory requirements. The linear subsystem models - in most cases obtained from FE discretization - are reduced with special consideration of the interface degrees of freedom (DOFs) and for a given frequency range of interest in a first step. Then, the reduced substructure models are assembled to the overall structural dynamics that has significantly less degrees of freedom. After analysis of this reduced model, the solution is expanded and the original vector of the physical DOFs is recovered.

In the following, displacement-based CMS is performed, i.e. approximate solutions in a reduced subspace (the range of the rectangular matrix $\Theta$ ) are sought in a Rayleigh-Ritz sense,

$$
\boldsymbol{x} \approx \Theta \boldsymbol{q}
$$

where the vector $\boldsymbol{x}$ contains the $N$ unknown physical displacements and rotations and the vector $\boldsymbol{q}$ the $m<<N$ reduced modal coordinates. The matrix $\Theta$ is denoted as the reduction base, Ritz vector base or modal base. 


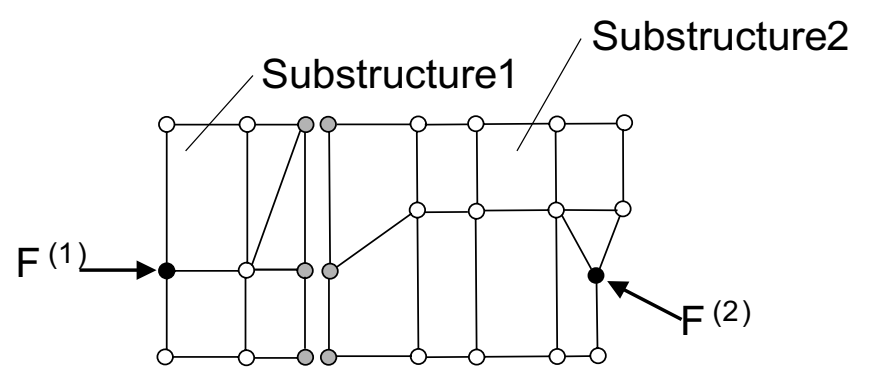

Fig. 1. Sketch of two substructures with marked interface degrees of freedom (DOF).

Typical methods combine normal modes of components obtained by different imposed boundary conditions at the interfaces (free, fixed or mass-loaded) and static modes from the static solution for applied interface loads (attachment modes) or imposed boundary displacements (constraint modes). Generally, the selection of the appropriate reduction base is performed in view of linear independence and completeness, low computational expense in their generation, automatic selection of their number and good convergence of the obtained solution to the exact (full) solution [3]. In the following, model reduction techniques are derived using substructure techniques as depicted in Fig. 1. Thereby, linear substructures are assumed, i.e. small deformations and linear elasticity.

\section{CMS methods for structures with friction}

In the following, two connected substructures with friction and nonlinear normal contact are considered, e.g. structures that arise from bolted members or jointed parts.

$$
\begin{aligned}
& {\left[\begin{array}{cc}
\mathbf{M}^{(1)} & \mathbf{0} \\
\mathbf{0} & \mathbf{M}^{(2)}
\end{array}\right]\left[\begin{array}{l}
\ddot{\boldsymbol{x}}^{(1)} \\
\ddot{\boldsymbol{x}}^{(2)}
\end{array}\right]+\left[\begin{array}{cc}
\mathbf{K}^{(1)} & \mathbf{0} \\
\mathbf{0} & \mathbf{K}^{(2)}
\end{array}\right]\left[\begin{array}{l}
\boldsymbol{x}^{(1)} \\
\boldsymbol{x}^{(2)}
\end{array}\right]+\left[\begin{array}{l}
\mathbf{B}_{\mathrm{T}}^{(1)} \\
\mathbf{B}_{\mathrm{T}}^{(2)}
\end{array}\right] \boldsymbol{F}_{\mathrm{T}}^{\mathrm{c}}+\left[\begin{array}{l}
\mathbf{B}_{\mathrm{N}}^{(1)} \\
\mathbf{B}_{\mathrm{N}}^{(2)}
\end{array}\right] \boldsymbol{F}_{\mathrm{N}}^{\mathrm{c}}=\boldsymbol{F}_{\text {ext }}} \\
& \dot{\boldsymbol{F}}_{\mathrm{T}}^{\mathrm{c}}=\boldsymbol{f}_{\mathrm{T}}^{\mathrm{c}}\left(\boldsymbol{F}_{\mathrm{T}}, \boldsymbol{x}_{\mathrm{rel}}, \boldsymbol{F}_{\mathrm{N}}^{\mathrm{c}}, \boldsymbol{\mu}, \boldsymbol{c}_{\mathrm{T}}\right), \boldsymbol{x}_{\mathrm{rel}}=\mathbf{B}_{\mathrm{T}}^{(1)^{\mathrm{T}}} \boldsymbol{x}^{(1)}-\mathbf{B}_{\mathrm{T}}^{(2)^{\mathrm{T}}} \boldsymbol{x}^{(2)} \\
& \boldsymbol{F}_{\mathrm{N}}^{\mathrm{c}}=\boldsymbol{f}_{\mathrm{N}} \underbrace{\mathbf{B}_{\mathrm{N}}^{(1)^{\mathrm{T}}} \boldsymbol{x}^{(1)}-\mathbf{B}_{\mathrm{N}}^{(2)^{\mathrm{T}}} \boldsymbol{x}^{(2)}}_{\boldsymbol{g}}, \boldsymbol{c}_{\mathrm{N}, 0}, \boldsymbol{c}_{\mathrm{N}, 1}) .
\end{aligned}
$$

The dynamics of the linear substructures 1 and 2 are coupled by the nodal tangential forces $\boldsymbol{F}_{\mathrm{T}}^{\mathrm{c}}$ and nodal normal forces $\boldsymbol{F}_{\mathrm{N}}^{\mathrm{c}}$. The matrices $\mathbf{B}_{\mathrm{T}}^{(i)}$ and $\mathbf{B}_{\mathrm{N}}^{(i)}$ capture on which physical DOFs the forces in the joint interface act. They are coincidence matrices for the full-order model if no reduction is applied. The tangential forces are obtained by appropriate discretization of the friction laws including the vector of the relative displacements $\boldsymbol{x}_{\text {rel }}$, whereas the normal forces are obtained from a nonlinear contact law including the relative gap $\boldsymbol{g}$ in normal direction. For the friction, the chosen model can be thought of an elastic-plastic model is chosen (similar to a Jenkins element in 1-D) which can be written as an evolution equation for the coupled friction forces in the local $x$ and $y$ directions. For the contact law, a nonlinear pressure-gap relationship models separation (no tension) and an increase in stiffness for increased penetration, see Fig. 2. Please note that Eq. 2 is a general representation, valid for a broad class of friction and contact models, although the special properties of the applied friction model are later exploited in the numerical implementation.

\subsection{Craig-Bampton method}

The Craig-Bampton method [4] is shortly presented starting from the structural dynamics of a linear substructure $k$,

$$
\mathbf{M} \ddot{\boldsymbol{x}}+\mathbf{K} \boldsymbol{x}=\boldsymbol{F},
$$




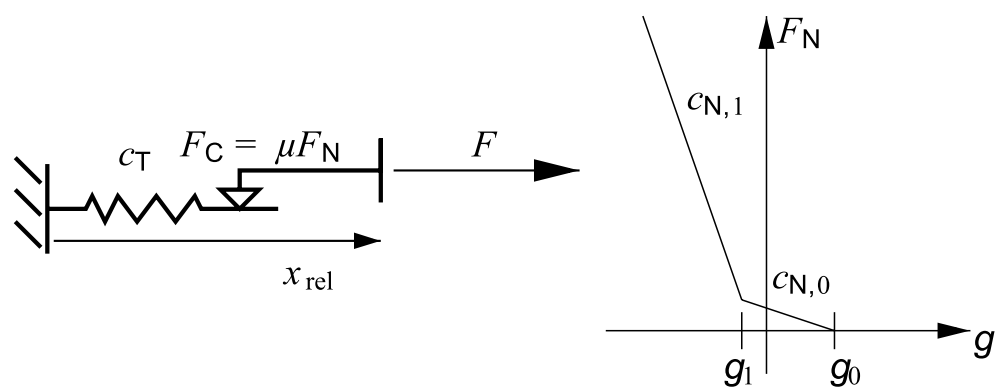

Fig. 2. Left: Elasto-plastic friction model (shown for 1-D case); Right: Nonlinear contact law in normal direction.

which is partitioned into free (inner) and interface DOFs. Since all quantities are related to a substructure $k$, the index is omitted for brevity. The DOFs are transformed to interface coordinates $\boldsymbol{x}_{i}$ and additional relative coordinates $\boldsymbol{x}_{\mathrm{r}}$,

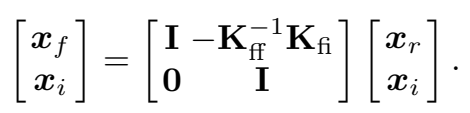

The interface DOFs are kept as physical coordinates, whereas the coordinates $\boldsymbol{x}_{\mathrm{r}}$ are reduced according to the Craig-Bampton method [4] as described in the following. The eigenvalue problem of

$$
\mathbf{M}_{\mathrm{ff}} \ddot{\boldsymbol{x}}_{\mathrm{r}}+\mathbf{K}_{\mathrm{ff}} \boldsymbol{x}_{\mathrm{r}}=0
$$

gives the first $m_{\mathrm{r}}$ fixed interface modes stacked in the modal base $\Theta_{\mathrm{r}}$. Thereby, the choice of the number $m_{\mathrm{r}}$ of retained modes depends on the desired dynamic bandwidth for which accuracy is demanded. Finally, with the approximation of the relative coordinates, $\boldsymbol{x}_{\mathrm{r}} \approx \Theta_{\mathrm{r}} \boldsymbol{x}_{\mathrm{m}}$, it follows

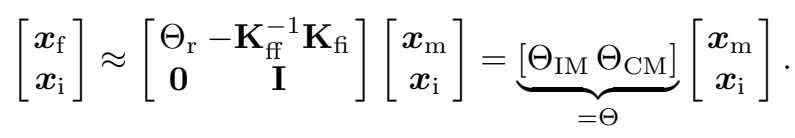

The modal base consists of two parts, the fixed-interface modes $\Theta_{\mathrm{IM}}$ and the constraint modes $\Theta_{\mathrm{CM}}$, which are the static solution for unit displacement of each individual interface DOF when the other interface DOFs are fixed. The reduced matrices are found by projection of the system matrices on the reduction base,

$$
\hat{\mathbf{K}}=\Theta^{\mathrm{T}} \mathbf{K} \Theta, \hat{\mathbf{M}}=\Theta^{\mathrm{T}} \mathbf{M} \Theta .
$$

Note that the obtained reduced mass and stiffness matrices are dense matrices whereas the original matrices are sparse. The reduction is performed for each substructure $k=\{1,2\}$ individually, where the interface DOFs are the DOFs of the joint interface of the substructure. Because of the similarity of the assembly step to the FE method, the reduced substructures can also be seen as superelements.

\subsection{Craig-Bampton reduction approach with common interface reduction (Method A)}

For further order reduction, the found constraint mode bases can be reduced (so-called interface reduction). As a novel way, both interface DOFs are reduced for the overall structure in one step. This allows to first reduce all substructures by the Craig-Bampton method and the interface DOFs later when the overall structure model is assembled. As an advantage, the number of retained interface DOFs can be distributed between the separate substructures in an optimal sense.

Each reduction base $\Theta^{(k)}$ consists of a normal mode base $\Theta_{\mathrm{NM}}^{(k)}$ and a constraint mode base $\Theta_{\mathrm{CM}}^{(k)}$, i.e.

$$
\Theta^{(k)}=\left[\Theta_{\mathrm{NM}}^{(k)}, \Theta_{\mathrm{CM}}^{(k)}\right] .
$$

Fixed-interface modes are used following the Craig-Bampton approach, i.e. $\Theta_{\mathrm{NM}}=\Theta_{\mathrm{IM}}$. A static condensation on the joint interface DOFs is performed for both interfaces by using 


$$
\Theta_{\mathrm{s}}=\left[\begin{array}{cc}
\Theta_{\mathrm{CM}}^{(1)} & \mathbf{0} \\
\mathbf{0} & \Theta_{\mathrm{CM}}^{(2)}
\end{array}\right]
$$

which gives the reduced system matrices

$$
\hat{\mathbf{M}}_{\mathrm{s}}=\Theta_{\mathrm{s}}^{\mathrm{T}}\left[\begin{array}{cc}
\mathbf{M}^{(1)} & \mathbf{0} \\
\mathbf{0} & \mathbf{M}^{(2)}
\end{array}\right] \Theta_{\mathrm{s}} \text { and } \hat{\mathbf{K}}_{\mathrm{s}}=\Theta_{\mathrm{s}}^{\mathrm{T}}\left[\begin{array}{cc}
\mathbf{K}^{(1)} & \mathbf{0} \\
\mathbf{0} & \mathbf{K}^{(2)}
\end{array}\right] \Theta_{\mathrm{s}} .
$$

Now, a reduced eigenvalue problem on the interface is formulated,

$$
\left(\hat{\mathbf{K}}_{\mathrm{s}}-\omega^{2} \hat{\mathbf{M}}_{\mathrm{s}}\right) \boldsymbol{\psi}=\mathbf{o}
$$

and solved for the desired number $m_{\mathrm{i}}$ of eigenvectors for the overall structure. Expansion to the substructure DOF vectors yields the reduced constraint-mode base $\Theta_{\mathrm{CM}}^{*}$ that is combined with normal mode base of the overall structure,

$$
\Theta=\left[\Theta_{\mathrm{NM}}, \Theta_{\mathrm{CM}}^{*}\right] .
$$

Then, the common reduction base $\Theta$ is applied to the mass and stiffness matrices, i.e.

$$
\hat{\mathbf{M}}=\Theta^{\mathrm{T}}\left[\begin{array}{cc}
\mathbf{M}^{(1)} & \mathbf{0} \\
\mathbf{0} & \mathbf{M}^{(2)}
\end{array}\right] \Theta \text { and } \hat{\mathbf{K}}=\Theta^{\mathrm{T}}\left[\begin{array}{cc}
\mathbf{K}^{(1)} & \mathbf{0} \\
\mathbf{0} & \mathbf{K}^{(2)}
\end{array}\right] \Theta .
$$

For completeness, $\Theta^{\mathrm{T}}$ is multiplied with the nonlinear forces as well as with the external forces of Eq. (2).

\subsection{Free-interface reduction approach with reduced set of joint constrained interface modes (Method B)}

The following reduction methodology is motivated by a publication of [18] where the law of action and reaction at the joint interface is exploited in order to introduce Joint Interface Modes. This is now applied to systems with friction.

Starting again from the structural dynamics of the linear substructure $k$, free interface modes are calculated by the eigenvalue problem

$$
\left(\mathbf{K}^{(k)}-\omega^{2} \mathbf{M}^{(k)}\right) \boldsymbol{\psi}=\mathbf{o}
$$

Then, a modal base is constructed by the combination of the $m$ free interface normal modes with the lowest eigenfrequencies to capture the system dynamics, $\Theta_{\mathrm{NM}}^{(k)}$, and the constraint modes $\Theta_{\mathrm{CM}}^{(k)}$ from Eq. (8). After static condensation on the interface DOFs similar to Eq. (12), the obtained system is additionally partitioned with respect to the substructures of the joint DOFs. This gives the reduced local system

$$
\left[\begin{array}{ll}
\hat{\boldsymbol{M}}_{\mathrm{i}}^{(11)} & \hat{\boldsymbol{M}}_{\mathrm{i}}^{(12)} \\
\hat{\boldsymbol{M}}_{\mathrm{i}}^{(12)} & \hat{\boldsymbol{M}}_{\mathrm{i}}^{(22)}
\end{array}\right]\left[\begin{array}{l}
\ddot{\boldsymbol{x}}_{\mathrm{i}}^{(1)} \\
\ddot{\boldsymbol{x}}_{\mathrm{i}}^{(2)}
\end{array}\right]+\left[\begin{array}{ll}
\hat{\boldsymbol{K}}_{\mathrm{i}}^{(11)} & \hat{\boldsymbol{K}}_{\mathrm{i}}^{(12)} \\
\hat{\boldsymbol{K}}_{\mathrm{i}}^{(12)} & \hat{\boldsymbol{K}}_{\mathrm{i}}^{(22)}
\end{array}\right]\left[\begin{array}{l}
\boldsymbol{x}_{\mathrm{i}}^{(1)} \\
\boldsymbol{x}_{\mathrm{i}}^{(2)}
\end{array}\right]=\left[\begin{array}{l}
\boldsymbol{F}_{\mathrm{i}}^{(1)} \\
\boldsymbol{F}_{\mathrm{i}}^{(2)}
\end{array}\right]=\left[\begin{array}{c}
\boldsymbol{F}_{\mathrm{i}}^{(1)} \\
-\boldsymbol{F}_{\mathrm{i}}^{(1)}
\end{array}\right] .
$$

If the friction interface is located between two completely separated substructures in this work, the coupling matrices evaluate to zero (this important property is not mentioned in [18]), i.e.

$$
\hat{\mathbf{M}}_{\mathrm{i}}^{(12)}=0, \hat{\mathbf{K}}_{\mathrm{i}}^{(12)}=0 .
$$

Neglection of the inertia effects in Eq. (17) allows to express $\boldsymbol{x}_{\mathrm{i}}^{(1)}$ by $\boldsymbol{x}_{\mathrm{i}}^{(2)}$

$$
\boldsymbol{x}_{\mathrm{i}}^{(1)}=\underbrace{-\left(\hat{\boldsymbol{K}}_{\mathrm{i}}^{(11)}+\hat{\boldsymbol{K}}_{\mathrm{i}}^{(12)}\right)^{-1}\left(\hat{\boldsymbol{K}}_{\mathrm{i}}^{(22)}+\hat{\boldsymbol{K}}_{\mathrm{i}}^{(12)}\right)}_{=\mathbf{G}} \boldsymbol{x}_{\mathrm{i}}^{(2)},
$$

which is plugged back into Eq. (17). The obtained modified reduced local system dynamics leads again to an eigenvalue problem,

$$
\left(\hat{\mathbf{K}}-\omega^{2} \hat{\mathbf{M}}\right) \psi=\mathbf{o}
$$


Table 1

Material and geometric properties of the investigated struc-

ture

\begin{tabular}{lcc}
\hline Parameter & Beam structure & Friction damper \\
\hline length & $775 \mathrm{~mm}$ & $160 \mathrm{~mm}$ \\
width & $40 \mathrm{~mm}$ & $40 \mathrm{~mm}$ \\
Thickness & $3 \mathrm{~mm}$ & $3 \mathrm{~mm}$ \\
material & steel & steel \\
Young's modulus & $205 \mathrm{GPa}$ & $205 \mathrm{GPa}$ \\
Poisson's ratio & 0.3 & 0.3 \\
density & $8000 \mathrm{~kg} / \mathrm{m}^{3}$ & $8000 \mathrm{~kg} / \mathrm{m}^{3}$ \\
\hline
\end{tabular}

which is solved for the desired number $m_{\mathrm{j}}$ of lowest eigenvectors for the overall structure. Expansion of this vectors to the substructure DOF vector gives the reduced constraint-mode bases for $m_{\mathrm{j}}$ joint interface modes,

$$
\Theta_{\mathrm{CM}}^{(2)}{ }^{*}=\left[\begin{array}{cc}
-\hat{\boldsymbol{K}}_{\mathrm{ff}}^{(2)^{-1}} \hat{\boldsymbol{K}}_{\mathrm{fi}}^{(1)} & \mathbf{I}
\end{array}\right] \underbrace{\left[\hat{\boldsymbol{\psi}}_{1}, \ldots, \hat{\boldsymbol{\psi}}_{m_{\mathrm{j}}}\right]}_{=\hat{\Theta}_{\mathrm{i}}}, \Theta_{\mathrm{CM}}^{(1)}{ }^{*}=\left[\begin{array}{c}
-\hat{\boldsymbol{K}}_{\mathrm{ff}}^{(1)-1} \hat{\mathbf{K}}_{\mathrm{fi}}^{(1)} \mathbf{G} \\
\mathbf{G}
\end{array}\right] \hat{\Theta}_{\mathrm{i}} .
$$

Finally, the derived base $\Theta_{\text {IM }}$ is added to the reduction base according to Eq. (10),

$$
\Theta^{*}=\left[\begin{array}{ccc}
\Theta_{\mathrm{NM}}^{(1)} & \mathbf{0} & \Theta_{\mathrm{CM}}^{(1)^{*}} \\
\mathbf{0} & \Theta_{\mathrm{NM}}^{(2)} & \Theta_{\mathrm{CM}}^{(2)}
\end{array}\right]
$$

\subsection{Static attachment mode}

For the interface reduction shown up to now, the nodes where the applied clamping forces act are not taken into consideration in the model reduction. This leads to the 10\% remaining error visible in Fig. 4. In a second step, an attachment mode for the applied clamping load is added to the reduction base, which guarantees zero model reduction error for the static case,

$$
\Theta=\left[\Theta^{*}, \boldsymbol{x}_{\text {static }}\right] \text {. }
$$

\section{Application: Prediction of friction damping by transient simulations}

For linear structures, the main criteria in the application of model reduction and substructuring methods is the accurate prediction of eigenfrequencies and eigenvectors, whereas the prediction of damping in joints additionally demands accurate prediction of the normal pressure. Hence, the error of the contact pressure should be specifically evaluated in order to make sure that correct damping prediction is possible.

\subsection{Investigated test structure}

A beam with attached friction damper (see Table 1 for dimensions and Fig. 3 for FE mesh) is used to investigate the proposed reduction methods. The bolts are modeled as discrete masses connected by shaft stiffnesses. The imposed pretension by the tightened screws is captured by special pretension elements.

\subsection{Convergence of the static solution}

For all considered reduction methods, the static convergence behavior is investigated as a preliminary for accurate damping prediction. Specifically, the convergence of the reduced model solution to the exact full order solution with 


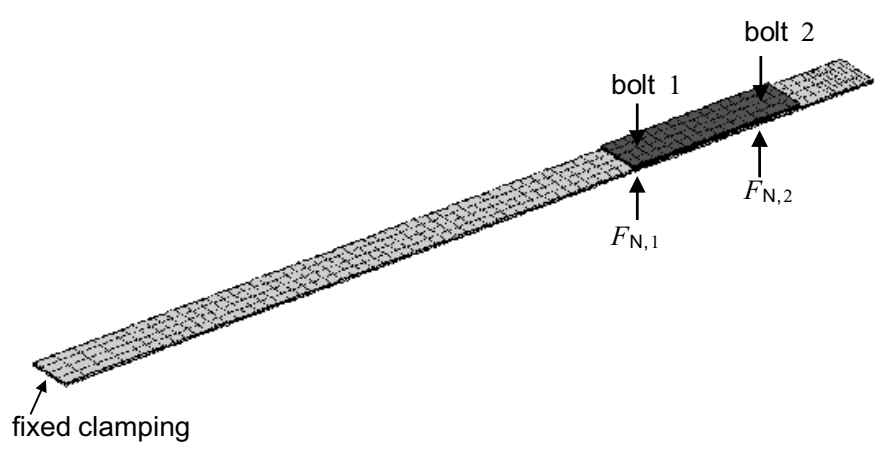

Fig. 3. FE mesh of test structure used for model reduction.

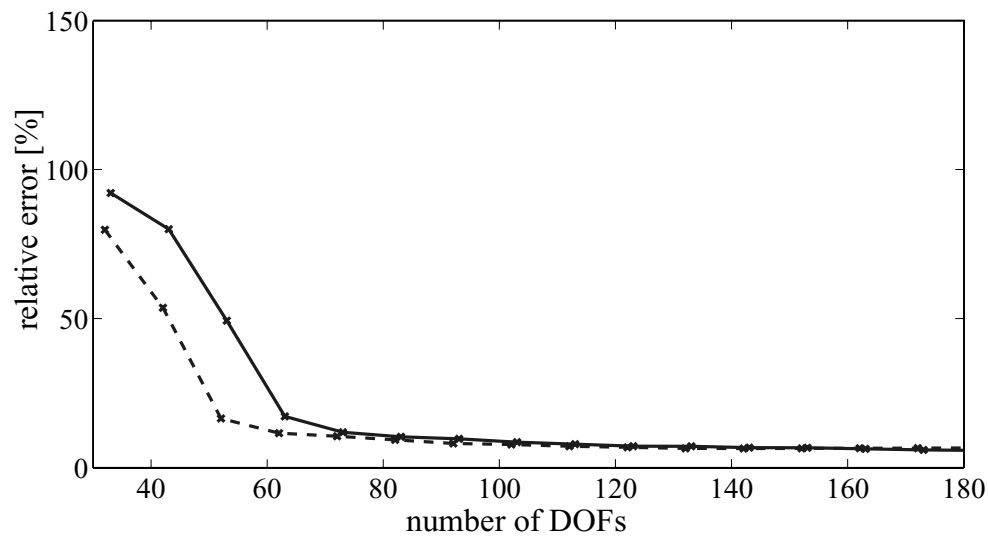

Fig. 4. Comparison of the static strain energy norm error $e_{\text {strain }}$ Eq. (24) for increasing number of (generalized) interface DOFs for method A $(-)$ and method B (- - ).

increasing number of retained generalized interface DOFs is evaluated. For that, all static loads are applied, i.e. the bolt pretension is modeled by prescribed normal forces $F_{\mathrm{N}, 1}$ and $F_{\mathrm{N}, 2}$.

If the strain energy norm is applied, the relative error is defined for the reduced model static solution $\boldsymbol{u}$ with respect to the strain energy $E$ composed of the linear parts and the contact and friction contributions

$$
e_{\text {strain }}=\frac{E-E_{\text {ref }}}{E_{\text {ref }}}
$$

It is calculated for each reduction method and a fixed number of normal modes for an increasing number of retained generalized interface DOFs, i.e. decreasing reduction level of the constraint modes. For this comparison, no additional static attachment modes are used. As seen in Fig. 4, strictly monotonic behavior is observed as expected and both methods show good convergence, although method B yields superior convergence for this specific problem for small DOF numbers in terms of all investigated error norms. For accurate damping prediction, the relative normal displacement error in the contact area is very important. Hence, the contact gap error is visualized for two example reduction degrees in Fig. 5 for method B. For higher accuracy of the reduced solution, the error is more equally distributed on the contact interface. Furthermore, due to the symmetry of the problem, the error profile is also symmetric.

\subsection{Frequency Response Functions (FRF)}

A set of accelerance frequency response functions (FRF) obtained from simulations of the exact full order model and of the reduced models are compared in Figs 6 and 7. The reduction method A is the Craig-Bampton method with 


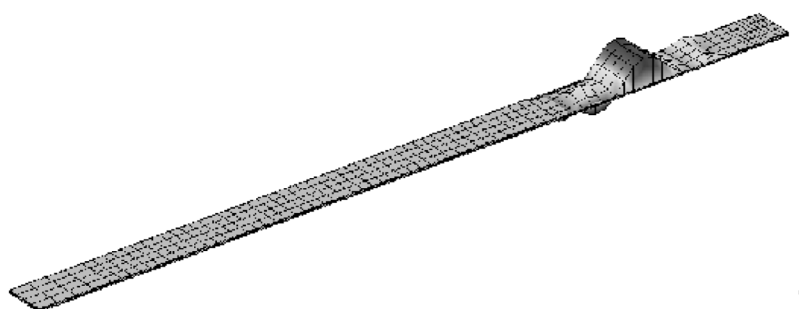

(a) $147 \%$ relative gap error (42 interface DOFs)

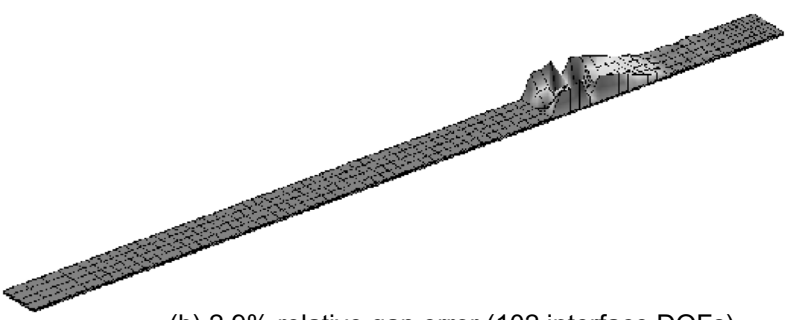

(b) $2.9 \%$ relative gap error (102 interface DOFs)

Fig. 5. Absolute gap error $g-g_{\text {ref }}$ (individually normalized) visualized as vertical displacements on the contact area of the FE model of the beam substructure (damper not shown, displacements outside of contact surface are set to 0 ).

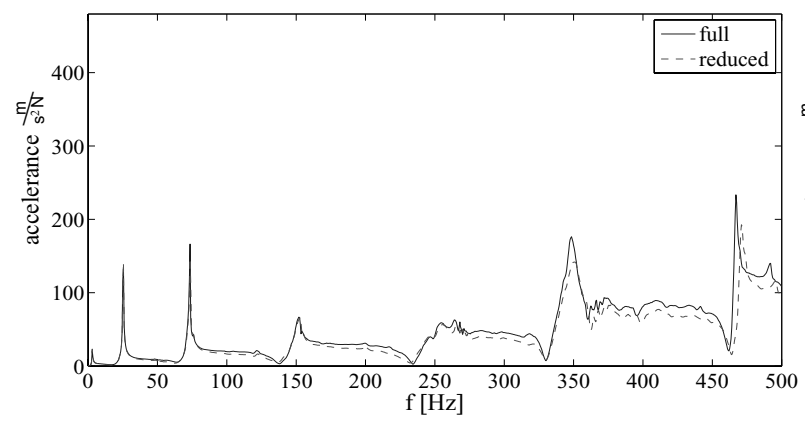

(a) $F_{\mathrm{N}, 1}=333 \mathrm{~N}$

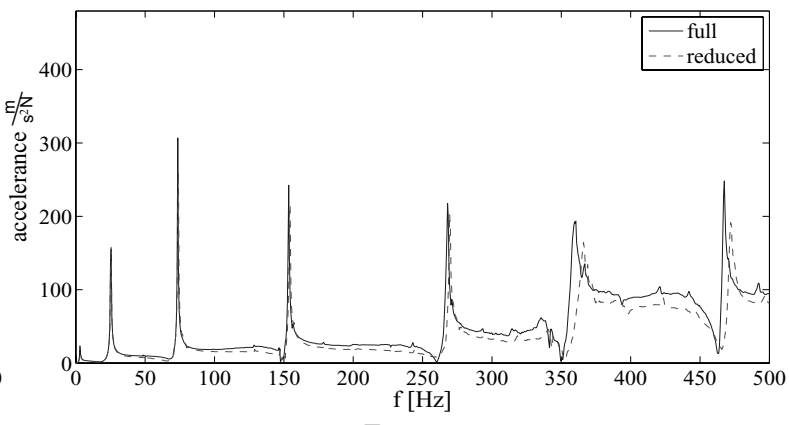

(b) $F_{\mathrm{N}, 1}=1000 \mathrm{~N}$

Fig. 6. FRFs for impulse excitation with method A for different normal forces (note the linear scale).

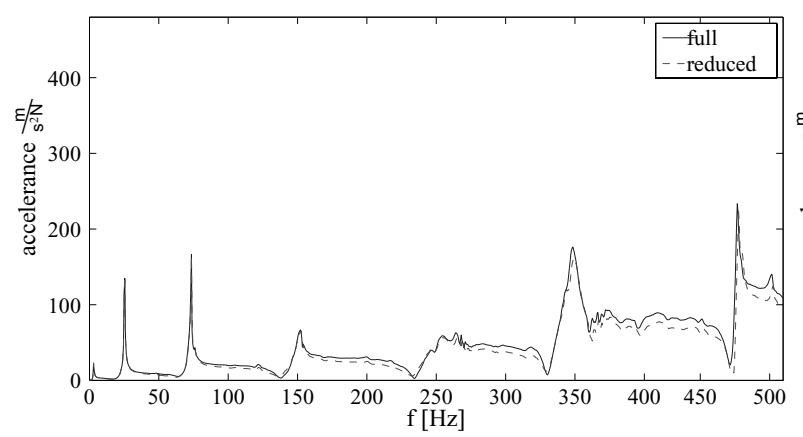

(a) $F_{\mathrm{N}, 1}=333 \mathrm{~N}$

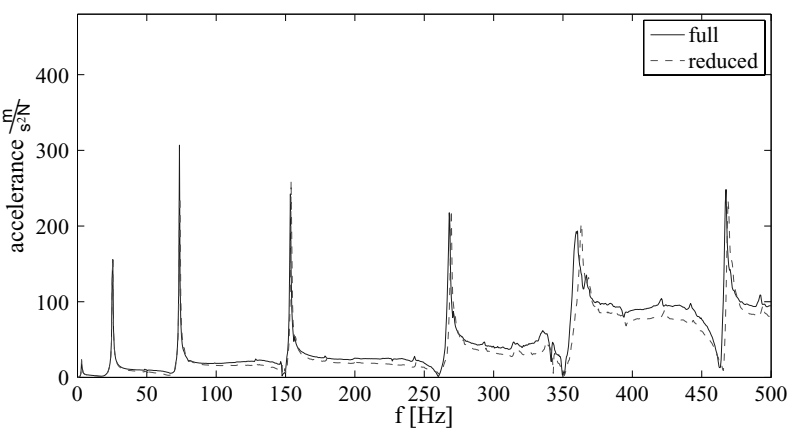

(b) $F_{\mathrm{N}, 1}=1000 \mathrm{~N}$

Fig. 7. FRFs for impulse excitation with method B for different normal forces (note the linear scale).

interface reduction for the whole structure with the exact nonlinear static solution added as additional attachment mode to the modal base. Method B is based on the free interface reduction approach with joint interface mode reduction. As in method $\mathrm{A}$, the exact nonlinear solution is added as an attachment mode. The FRFs are calculated from simulated transient responses of $2 \mathrm{~s}$ for experimentally measured impulse force excitation signals. In order to avoid leakage effects, an exponential window of $1 \mathrm{~s}$ time constant is applied. Normal forces $F_{\mathrm{N}, 1}$ of $333 \mathrm{~N}, 667 \mathrm{~N}$ and $1000 \mathrm{~N}$ are applied to the variable screw at bolted joint 1 whereas the other screw is fixed by a normal force $F_{\mathrm{N}, 2}=4000 \mathrm{~N}$. Example results for the two methods compared to the exact solution are presented in Figs 6 and 7. For comparable results, the method A needs 71 DOFs whereas the method B only needs 56 DOFs for the same or even better prediction accuracy, which is contributed to the faster convergence of the constraint mode reduction see Fig. 4.

The plots and the calculated errors show that the eigenfrequencies, the peak heights and widths (which are important for the damping prediction) as well as the transfer zeros are matched very well by the reduced models. 
Table 2

Evaluation of the computation times for the calculation of a transient response for $t_{\mathrm{sim}}=0.2 \mathrm{~s}$ simulation time to an impulse force excitation. Measured pure CPU times $t_{\mathrm{cpu}}$ on a standard PC using a single core (3 GHz Intel Xeon/Core 5160) are related to the simulation time. The values that are obtained for the experimentally determined contact parameters (Table 3) are marked with the asterisks

\begin{tabular}{lcc}
\hline Model & DOFs & Ratio $t_{\mathrm{cpu}} / t_{\mathrm{sim}}$ \\
\hline MATLAB $+C$ full model & 5955 & $20510^{*} \ldots 73553$ \\
ANSYS (full model) & 5955 & $37540 \ldots 59880$ \\
reduced model, standard Craig-Bampton & 533 & $73530^{*}$ \\
reduced model, with interface reduction & 73 & $709^{*}$ \\
reduced model, with interface reduction & 52 & $422^{*}$ \\
\hline
\end{tabular}

\subsection{Reduction of computation time}

In transient simulations of nonlinear systems, the number of DOFs has a strong impact on the computation time because nonlinear equilibrium iterations with updated tangential matrices must be performed at every time step. The nonlinear Newmark time integration scheme with Newton-Raphson iterations is applied using consistent tangential matrices. These iterations lead to very high computational cost which makes - in contrast to other CMS applications the initial effort to calculate the reduction base and to reduce the matrices vanish in the overall necessary effort for the analysis.

The found computation times for transient simulations of the full and differently reduced models are listed in Table 2, where the standard Craig-Bampton method without interface reduction step, the improved Craig-Bampton method A and the free-interface method B are compared to the original full model. In order to assess the required computation time, the ratio of necessary CPU time to simulation time is determined. Additionally, the found ratios are set in relation to the full order solution to obtain the improvement factors. For comparison reasons, the computation time for the full model with the commercial FE code ANSYS is given (using Newmark scheme and a line search algorithm). In view that its algorithms are implemented in a low-level programming language better optimized to the computer platforms in contrast to the MATLAB implementation of the presented solutions, i.e. in a high-level script programming language, the performance of the full order solution is considered as very good. Please note that ANSYS does not give detailed insight and control over the implemented algorithms, i.e. it is a somewhat black-box solution.

Both presented reduction methods yield excellent accuracy and hereby make a complete analysis on a standard $\mathrm{PC}$ feasible in a reasonable time. The required computation time for the standard Craig-Bampton method is found to be rather high because the reduction of the number of DOFs is compensated by the extra effort for the calculations with the dense system matrices.

\subsection{Simulation and analysis framework}

A simulation and analysis framework shown in Fig. 8 has been developed that is capable to use linear substructure models from commercial FE codes. The substructures may be additionally moved in space and are then assembled with the zero-thickness elements implementing the contact and friction effects on the defined contact areas in an assembly step [12]. Furthermore, reduction methods may be applied to built the overall structural dynamics or individual parts of it. Static and transient solvers are implemented for both full-order and reduced models and the results can be post-processed. One typical way of post-processing is to calculate frequency response function (FRF) and to estimate the modal parameters or to export the FRFs to commercial post-processing tools for special analysis.

\section{Experimental verification}

The developed efficient simulation framework employing model reduction is a key tool in order to perform model updating or parameter variations on a standard PC. In the sequel, results from such an updating procedure of a FE 


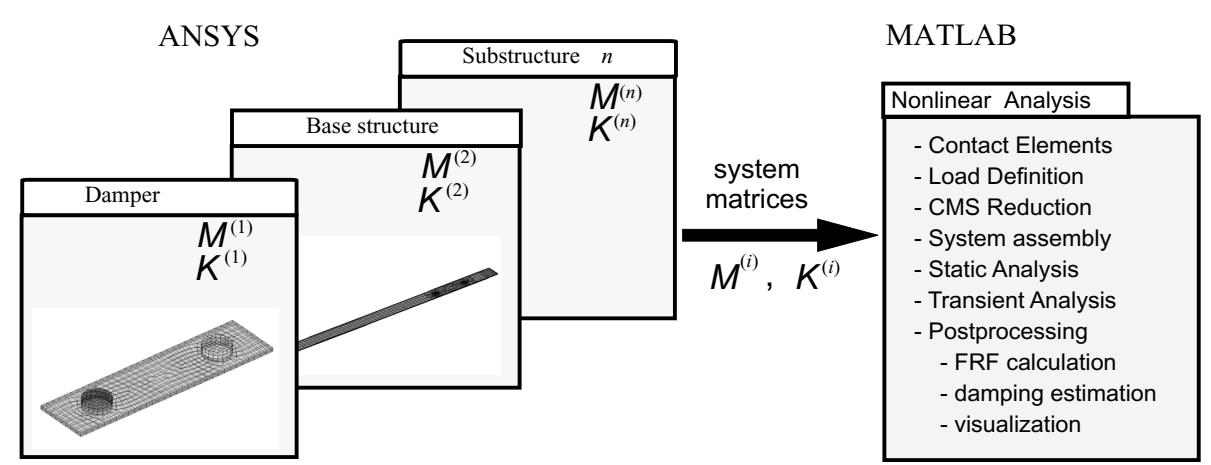

Fig. 8. Simulation framework for nonlinear structural analysis of mechanical structures with friction.

Fig. 9. Mesh of investigated structure with attached friction damper (example deformation and undeformed reference).

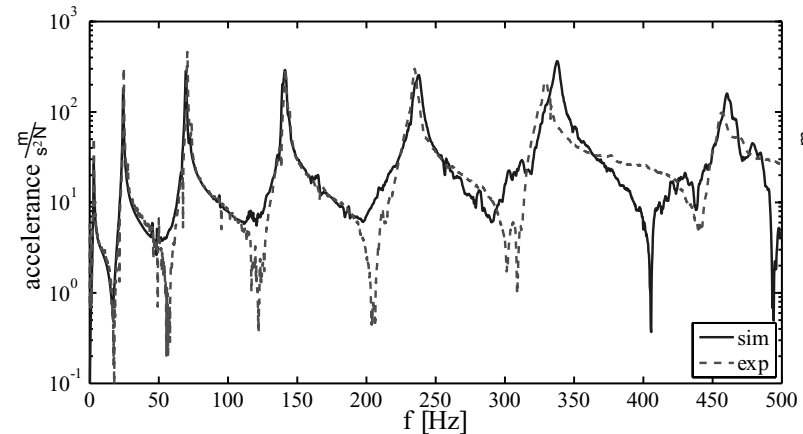

(a) $F_{\mathrm{N}, 1}=13 \mathrm{~N}$

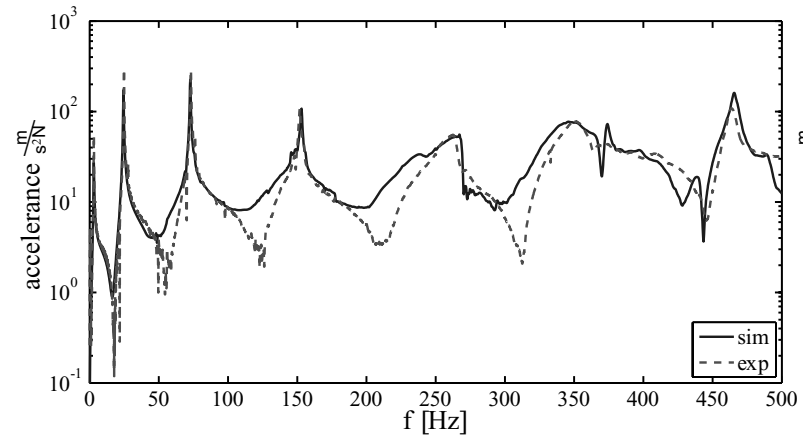

(c) $F_{\mathrm{N}, 1}=667 \mathrm{~N}$

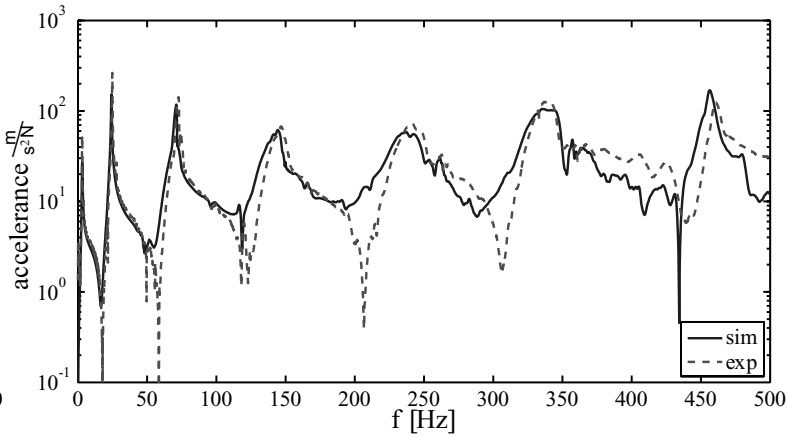

(b) $F_{\mathrm{N}, 1}=333 \mathrm{~N}$

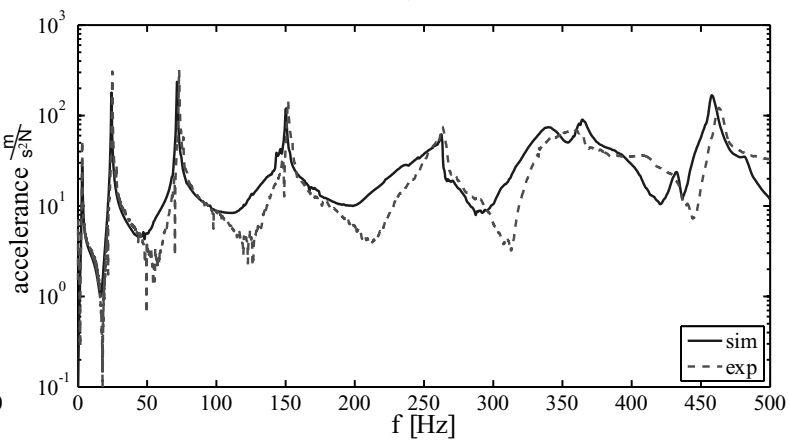

(d) $F_{\mathrm{N}, 1}=1000 \mathrm{~N}$

Fig. 10. Comparison of measured and simulated FRFs for best match parameter case (cf. Table 3). 
Table 3

Determined contact parameters for best match of simulated with experimental FRFs

\begin{tabular}{lcc}
\hline Parameter & Variable & Value \\
\hline friction coefficient & $\mu$ & 0.2 \\
normal stiffnesses & $c_{\mathrm{N}, 0}, c_{\mathrm{N}, 1}$ & $1.25 \cdot 10^{11} \frac{\mathrm{N}}{\mathrm{m}}, 2 \cdot 10^{12} \frac{\mathrm{N}}{\mathrm{m}}$ \\
gap distances & $g_{0}, g_{1}$ & $-1 \cdot 10^{-6} \mathrm{~m}, 0 \mathrm{~m}$ \\
tangential stiffness & $c_{\mathrm{T}}$ & $8 \cdot 10^{11} \frac{\mathrm{N}}{\mathrm{m}}$ \\
\hline
\end{tabular}

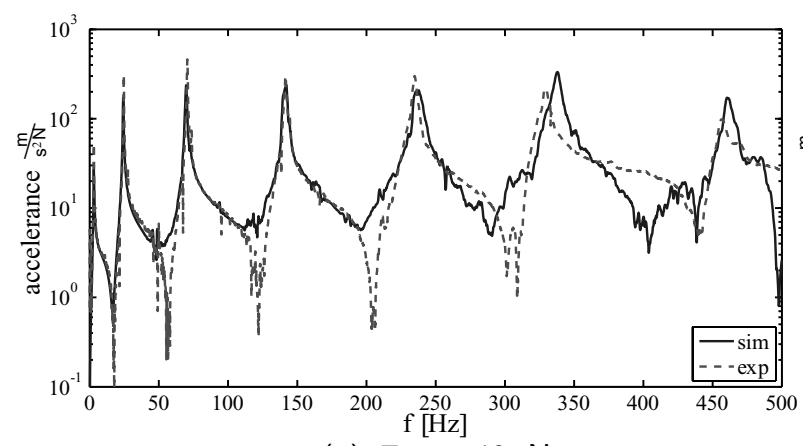

(a) $F_{\mathrm{N}, 1}=13 \mathrm{~N}$

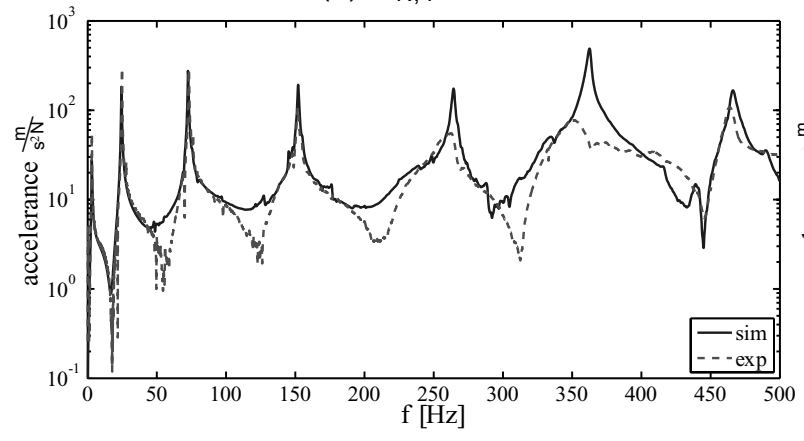

(c) $F_{\mathrm{N}, 1}=667 \mathrm{~N}$

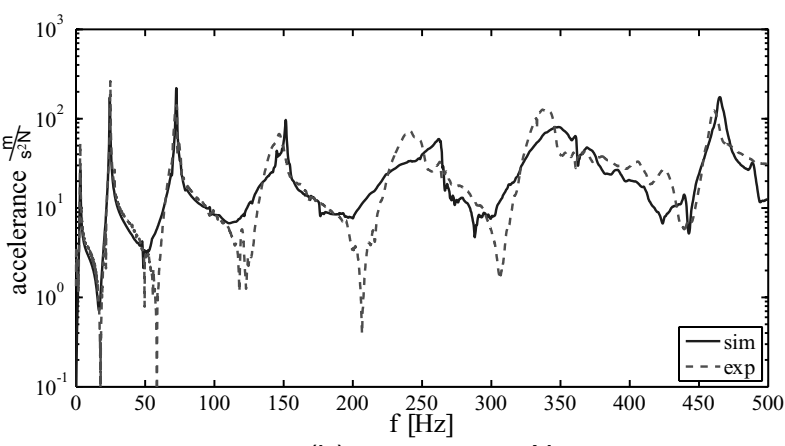

(b) $F_{\mathrm{N}, 1}=333 \mathrm{~N}$

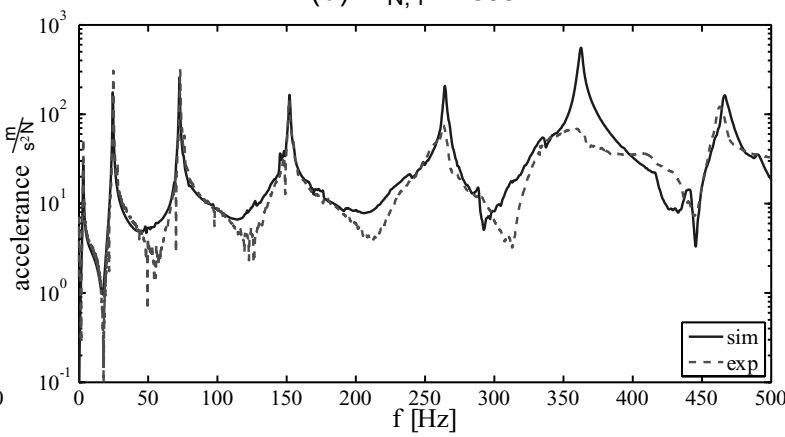

(d) $F_{\mathrm{N}, 1}=1000 \mathrm{~N}$

Fig. 11. Comparison of measured and simulated FRFs for with varied friction coefficient $\mu=0.4$.

model to experimental data are presented. For similar material parameters as given before in Table 1 for the model in Fig. 3, a refined FE model is used as shown in Fig. 9. Compared to the reduction test model, the bolts and holes are now modeled and the discretization is refined close to the bolts where high normal pressures appear. The FE model has now 21564 DOFs (603 pairs of contact nodes) and is reduced to 57 DOFs.

\subsection{Model updating}

For model updating, so-called linearizing excitation signals are employed because they yield linearized FRFs, i.e. FRFs resembling those obtained from linear systems [19]. Although random excitation is found to have best linearizing properties in experiments, deterministic impulse excitation is chosen because the necessary simulation time for random excitation would be very large. In the experimental setup, the excitation is applied by an impulse hammer close to the tip and the responses are measured at several points on the structure. The shown driving point FRFs are calculated from the measured acceleration on the opposite surface. The excitation is located on the mid-line of the beam, hence, virtually no torsional modes are excited. Measurements are conducted for a set of different normal forces $F_{\mathrm{N}, 1}$, whereas the normal force at the other damper end is again constantly set to $F_{\mathrm{N}, 2}=4000 \mathrm{~N}$. An exponential window of $2 \mathrm{~s}$ time constant is applied before the FRFs are calculated from five individual measurements averaged in the frequency domain. 


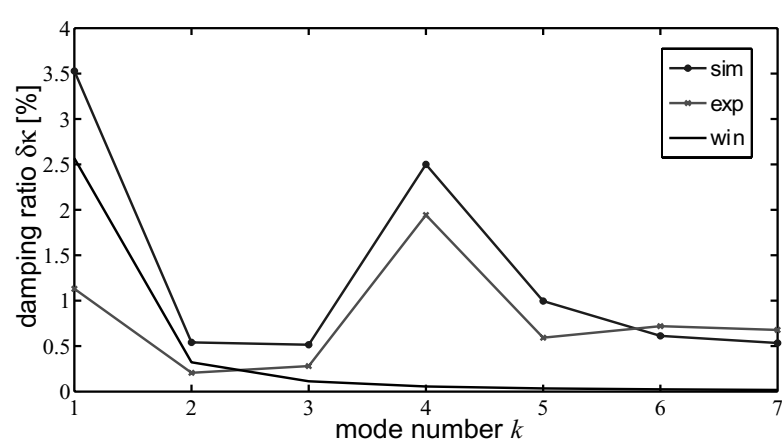

(a) $F_{\mathrm{N}, 1}=13 \mathrm{~N}$

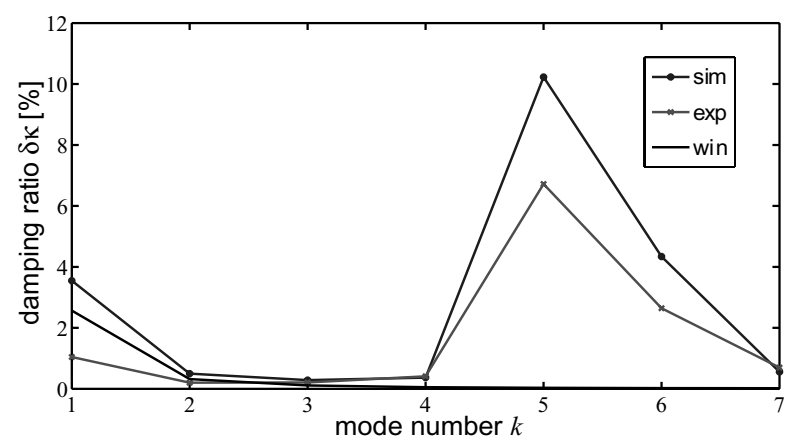

(c) $F_{\mathrm{N}, 1}=667 \mathrm{~N}$

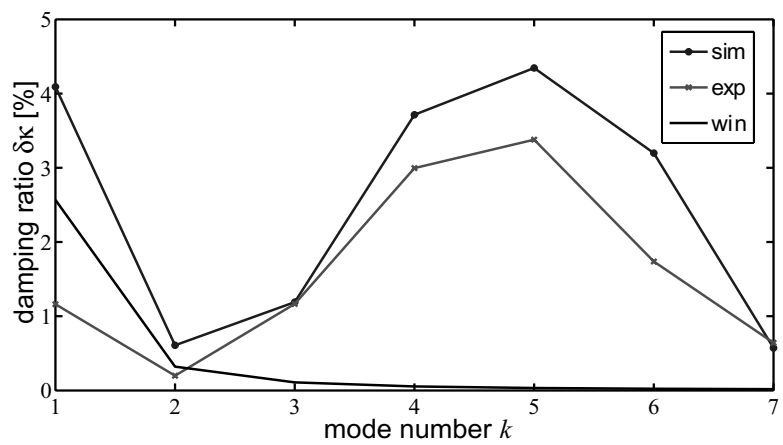

(b) $F_{\mathrm{N}, 1}=333 \mathrm{~N}$

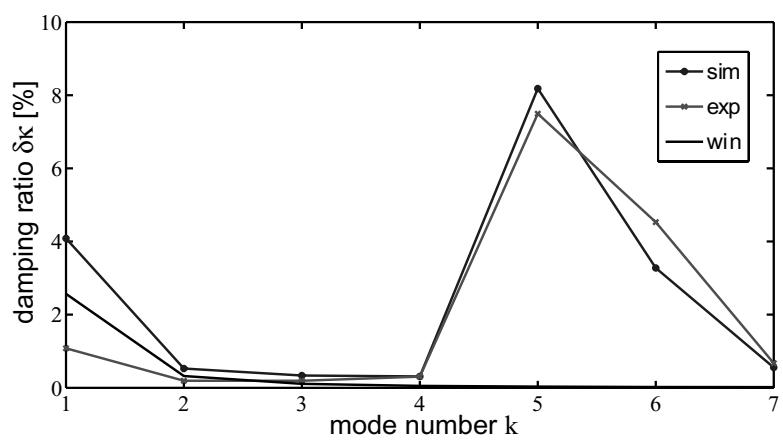

(d) $F_{\mathrm{N}, 1}=1000 \mathrm{~N}$

Fig. 12. Modal damping ratios $\delta_{k}$ from simulation and experiment (artificial window damping shown for information).

For the simulations, experimentally measured impulses are averaged and applied as loads. As in the measurements, FRFs are calculated from the $6 \mathrm{~s}$ responses using an exponential window of time constant $\tau=2 \mathrm{~s}$. Classical Rayleigh damping is assumed with parameters determined from experimental modal analysis of the base structure. In the model updating, the friction coefficient, the tangential contact stiffness and the normal contact stiffness are varied to match the experimental data to find the best match parameter set given in Table 3. For these parameters, the simulated and the measured FRFs are compared in Fig. 10. Obviously, the chosen excitation force with roughly $100 \mathrm{~N}$ peak force significantly excites higher harmonics of the system. Simulations have proven that they are mostly due to the normal contact between damper and base structure. Due to internal resonances, the amplitudes of these higher harmonics depend on their frequencies in relation to the resonance frequencies of the structure as well as on the spatial location of the friction and contact interface which makes them not predictable by analytical means. The qualitative shape of the resonance peaks, the width and height determining the damping and the locations of the higher harmonics are very well predicted by the simulations. Obviously, a very good match quality is observed for all measured normal force cases between $F_{\mathrm{N}, 1}=13$ and $F_{\mathrm{N}, 1}=1000$ N. Further results, presented in Fig. 11, show the effects of a higher friction coefficient of $\mu=0.4$.

\subsection{Evaluation of modal damping}

Although friction is a nonlinear effect, there is a big interest in evaluating the damping effect in terms of modal damping ratios known from linear structural dynamics. This is mainly because modal damping ratios can easily be measured and compared in engineering practice. For nonlinear structures - in contrast to linear ones - the excitation type and amplitude must be controlled for fair comparisons and correct analysis. The modal damping ratios are determined by the 3dB-bandwidth method from the FRFs [5]. As mentioned before, an exponential window is applied to the simulated or measured acceleration responses to prevent leakage. For the chosen exponential window with time constant $\tau$, the artificial damping $\delta_{k}^{\text {win }}$ added to the apparent modal damping $\delta_{k}^{\text {det }}$ of mode $k$ can be compensated for (under the assumption of linear dynamics) [5], i.e. $\delta_{k}=\delta_{k}^{\text {det }}-\delta_{k}^{\text {win }}$ with $\delta_{k}^{\text {win }}=1 /\left(2 \tau \omega_{k}\right)$. 
The modal damping ratios $\delta_{k}$ from simulations and experiments in Fig. 12 show excellent agreement. The numerically predicted ratios match the experimental ones quite well, especially if one keeps in mind that measurement errors influence much more the damping identification than the eigenfrequency identification. The variations in the damping of mode 1 is due to the high contribution of artificial window damping and violation of the linear dynamics assumption for the physical damping recovery. As expected, the found eigenfrequencies and the damping ratios strongly depend on the applied normal forces.

\section{Conclusions}

The usefulness of CMS methods applied to the simulation of mechanical structures with friction and joints has been demonstrated, by which parameter updating has become feasible for a real-world structure on a standard PC. Excellent agreement between the simulated and measured FRFs shows that the inclusion of friction in the design process is feasible to predict its influence on damping and eigenfrequencies as well as the generation of higher harmonics. As a strong advantage, the output variables of interest need not to be known before the reduction step, because the full displacement vector is recovered from the reduced solution. The developed own codes for the static and transient solvers offer the possibility to investigate various modifications of the contact model, to relatively easily incorporate it into optimization procedures or to simulate feedback controllers, that in general can not be implemented in commercial FE codes in a straight way [2].

Actual work focuses on applications to more complex structures of typical industrial applications. Furthermore, the semi-analytical harmonic balance method is employed for efficient damping calculation directly in the frequency domain for passively damped structures also exploiting the proposed model reduction techniques.

\section{References}

[1] H. Ahmadian, J.E. Mottershead, S. James, M. Friswell and C. Reece, Modelling and updating of large surface-to-surface joints in AWE-MACE structure, Mechanical Systems and Signal Processing 20 (2006), 868-880.

[2] J. Becker, Semi-Active Control of Friction Dampers and Feedforward Tracking Control Design for Structural Vibration Reduction, PhD thesis, Universität Stuttgart, 2009.

[3] R.R. Craig, A brief tutorial on substructure analysis and testing, In Proc. of IMAC XVIII (2000).

[4] R.J. Craig and M.C.C. Bampton, Coupling of substructures for dynamic analysis, AIAA Journal 6(7) (1968), 1313-1319.

[5] D.J. Ewins, Modal Testing: Theory and Practice, John Wiley \& Sons Inc., New York, 1984.

[6] L. Gaul, Wave transmission and energy dissipation at structural and machine joints, Journal of Vibration, Acoustics, Stress, and Reliability in Design 105 (1983), 489-496.

[7] L. Gaul, The influence of damping on waves and vibrations, Mechanical Systems and Signal Processing 13(1) (1999), 1-30.

[8] L. Gaul, H. Albrecht and J. Wirnitzer, Semi-active friction damping of large space truss structures, Journal of Shock and Vibration 11 (2004), 173-186.

[9] H. Jalali and H. Ahmadian, Identification of bolted lap joints parameters in assembled structures, Mechanical Systems and Signal Processing 21(2) (2007), 1041-1050.

[10] R.V. Kappagantu and B.F. Feeny, Part 1: Dynamical characterization of a frictionally excited beam, Nonlinear Dynamics 22(4) (2000), 317-333.

[11] R.V. Kappagantu and B.F. Feeny, Part 2: Proper orthogonal modal modeling of a frictionally excited beam, Nonlinear Dynamics 23(1) (2000), 1-11.

[12] M. Mayer and L. Gaul, Segment-to-segment contact elements for modelling joint interfaces in finite element analysis, Mechanical Systems and Signal Processing 21(2) (Feb. 2007), 724-734.

[13] C.H. Menq, J.H. Griffin and J. Bielak, The influence of microslip on vibratory response, part II: A comparison with experimental results, Journal of Sound and Vibration 107(2) (June 1986), 295-307.

[14] K. Popp, Nichtlineare Schwingungen mechanischer Strukturen mit Füge- oder Kontaktstellen, 74 74 3 (1994), $147-165$.

[15] Z.-Q. Qu, Model reduction for dynamical systems with local nonlinearities. AIAA Journal 40(2) (2002), 327-333.

[16] D.J. Segalman, Model reduction of systems with localized nonlinearities, J Comput Nonlinear Dynam 2(3) (July 2007), 249-266.

[17] H. Wentzel and M. Olsson, Numerical prediction of damping in structures with frictional joints, International Journal of Vehicle Noise and Vibration 2(2) (2006), 125-142.

[18] W. Witteveen and H. Irschik, Efficient modal formulation for vibration analysis of solid structures with bolted joints, In Proc. of IMAC XXV, Orlando, USA (2007).

[19] K. Worden and G.R. Tomlinson, Nonlinearity in Structural Dynamics, Institute of Physics Publishing, 2001. 

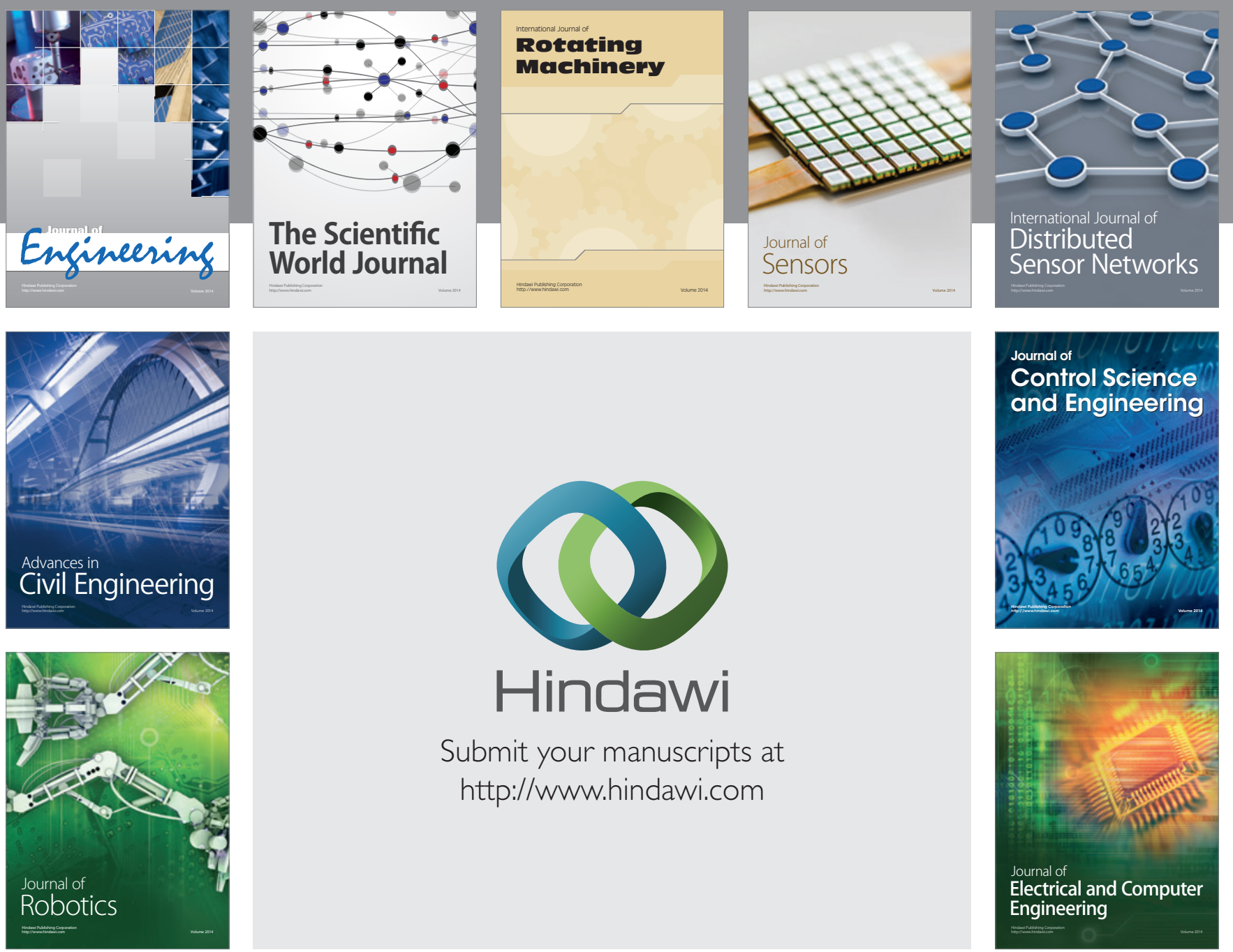

Submit your manuscripts at

http://www.hindawi.com
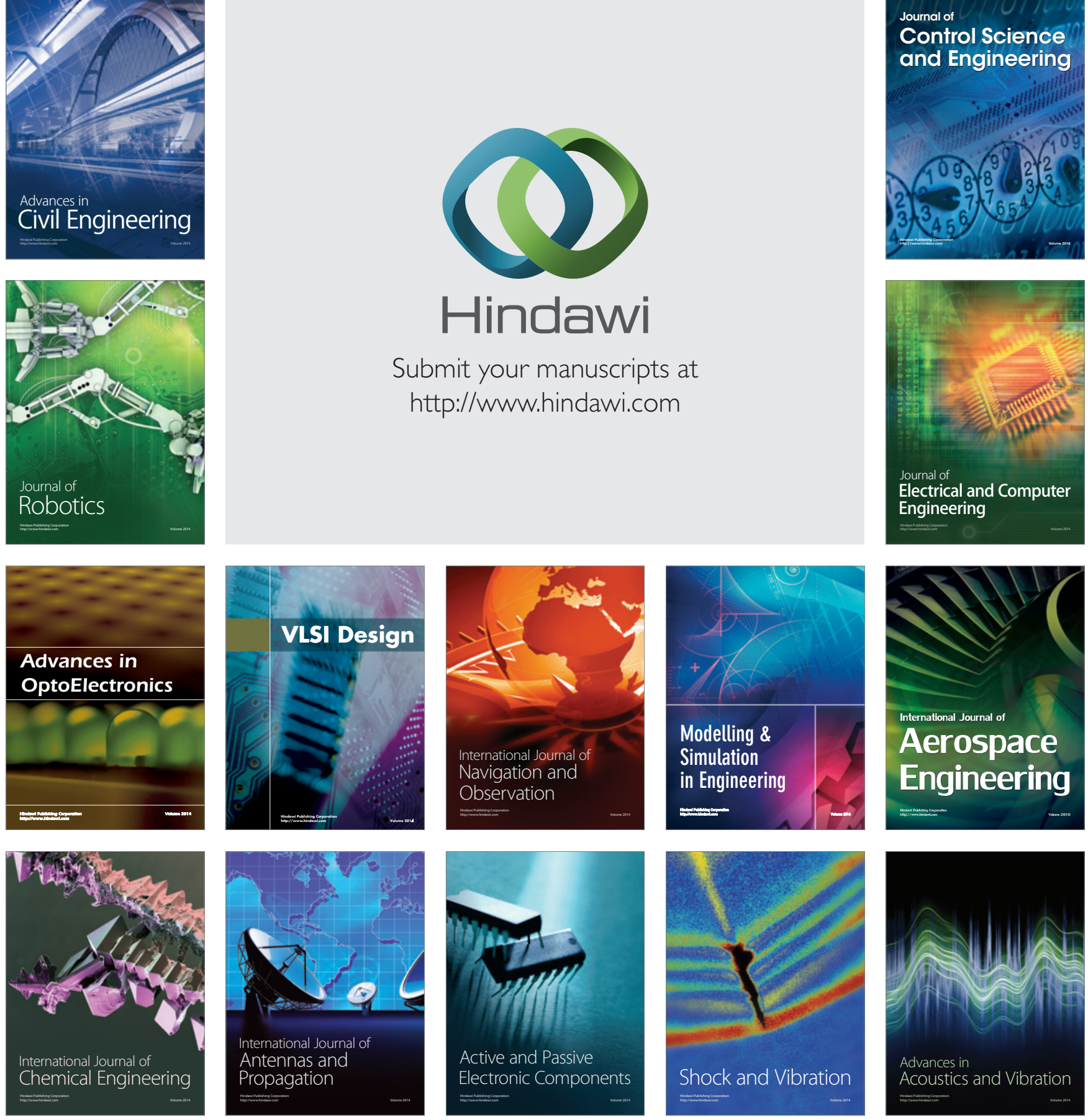